\title{
Indocyanine green fluorescence imaging for laparoscopic complex upper urinary tract reconstructions: a comparative study
}

\author{
Weijie Zhu ${ }^{1 \#}$, Shengwei Xiong ${ }^{1 \#}$, Yucai Wu ${ }^{1 \#}$, Dengxiang Zhang ${ }^{2}$, Chen Huang ${ }^{2}$, Han Hao ${ }^{1}$, Lei Zhang ${ }^{1}$, \\ Kunlin Yang ${ }^{1}$, Peng Zhang ${ }^{3}$, Hongjian Zhu $^{2}$, Xuesong Li $^{1}$, Liqun Zhou ${ }^{1}$ \\ ${ }^{1}$ Department of Urology, Peking University First Hospital, Institute of Urology, Peking University, National Urological Cancer Centre, Beijing, \\ China; ${ }^{2}$ Department of Urology, Beijing Jiangong Hospital, Beijing, China; ${ }^{3}$ Department of Urology, Emergency General Hospital, Beijing, China \\ Contributions: (I) Conception and design: L Zhou; (II) Administrative support: None; (III) Provision of study materials or patients: W Zhu, S Xiong; \\ (IV) Collection and assembly of data: W Zhu, S Xiong, Y Wu; (V) Data analysis and interpretation: P Zhang, Y Wu; (VI) Manuscript writing: All \\ authors; (VII) Final approval of manuscript: All authors. \\ \#These authors contributed equally to this work. \\ Correspondence to: Xuesong Li. Department of Urology, Peking University First Hospital, Institute of Urology, Peking University, National \\ Urological Cancer Centre, No. 8 Xishiku St, Xicheng District, Beijing 100034, China. Email: pineneedle@sina.com; Hongjian Zhu. Department of \\ Urology, Beijing Jiangong Hospital, No. 6 Rufuli St, Xicheng District, Beijing 100034, China. Email: hjzhu99@sina.com.
}

Background: To describe our technique for using an intraureteral injection of indocyanine green (ICG) and visualization under near-infrared fluorescence (NIRF) to facilitate challenging upper urinary tract reconstructions (UUTRs) and to present the comparative outcomes.

Methods: We collected 36 patients who underwent laparoscopic UUTRs between April 2019 and March 2020, and we divided the patients into two groups based on the use of ICG (ICG group and non-ICG group). Demographic characteristics, perioperative outcomes, and functional outcomes were compared between the two groups.

Results: There were 18 cases in the ICG group and 18 cases in the non-ICG group, respectively. There were no differences in the baseline characteristics between the two groups. The intraoperative time to identification of the ureter (TIU; $20.9 \pm 11.7$ vs. $30.0 \pm 14.6 \mathrm{~min}, \mathrm{P}=0.03$ ) and length of postoperative hospital stay (LPHS; $11.1 \pm 3.0$ vs. $16.6 \pm 10.0$ days, $\mathrm{P}=0.03$ ) were significantly shorter in the ICG group. There

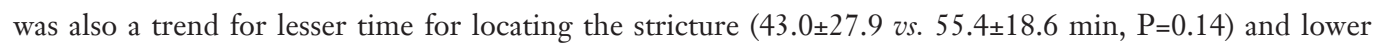
estimated blood loss (EBL) in the ICG group patients $(88.3 \pm 75.4$ vs. $91.7 \pm 46.2 \mathrm{~mL}, \mathrm{P}=0.22)$. During the mean 3.8-month follow-up for the ICG group and the 6.2-month for the non-ICG group, there was a trend for more severe complications in the non-ICG group.

Conclusions: Visualizing intraureteral ICG under NIRF is useful in challenging UUTRs, allows for rapid ureteral identification and accurate real-time delineation of the ureteral stricture margins, and provides encouraging follow-up outcomes compared with those in the non-ICG group.

Keywords: Indocyanine green (ICG); near-infrared fluorescence (NIRF); upper urinary tract reconstructions (UUTRs); minimal invasive surgery

Submitted Sep 12, 2020. Accepted for publication Jan 05, 2021.

doi: $10.21037 /$ tau-20-1261

View this article at: http://dx.doi.org/10.21037/tau-20-1261

\section{Introduction}

Upper urinary tract reconstructions (UUTRs) are usually complicated because of poor tissue planes, fibrosis, and anatomical abnormalities, which may be exacerbated by any previously attempted reparation. Key steps for successful UUTRs include resection of the obstructing and/or scarred segment, conservation of the healthy ureter, 
and establishment of a tension-free, water-tight, and wellvascularized anastomosis (1).

For UUTRs, minimally invasive treatment has been increasingly considered as an alternative to open surgery. A particularly critical issue in minimally invasive UUTRs is that unnecessary tissue manipulation or accidental trauma may cause ischemia and may compromise ureteropelvic or ureteral reparation. There is a lack of tactile feedback with laparoscopic instruments, although this may be offset by improvements in instrument dexterity and visualization (2).

Therefore, a technique that improves the ability of visual identification of the ureter and distinguishment of diseased tissues from a healthy ureter is very much needed. Indocyanine green (ICG) is a fluorescent dye; when it is activated by near infrared fluorescence (NIRF) light, it can visualize the desired anatomical structure in real time. As a real-time contrast agent, ICG is very suitable for intraoperative use because of its tissue penetrating ability, high signal-to-noise ratio, and excellent safety (3). ICG is established in dermatology, ophthalmology and vascular recognition in cardiology (4). It has been reported in urology literature that usage of ICG is helpful for sentinel lymph node dissection in radical prostatectomy; to distinguish between normal renal parenchyma and tumor and selective renal clamping in partial nephrectomy; and ICG in partial adrenalectomy to strengthen intraoperative tumor localization (5). And ICG has been utilized in UUTRs to assist in structural identification, perfusion assessment, and delineation of the margins as a real-time contrast agent. In the application of ICG in UUTRs, most of the articles are descriptive analysis to highlight the advantages of ICG (6). To date, there has been no comparative research on the use of this technology for laparoscopic UUTRs.

In this study, we compared the perioperative and followup outcomes between the ICG group and the non-ICG group in patients with lesions of the upper urinary tract (UUT), and we demonstrated that ICG may facilitate improved identification of the ureter, assessment of tissue vascularity, and excision of non-viable segments of the UUT. We present the following article in accordance with the STROBE reporting checklist (available at: http://dx.doi. org/10.21037/tau-20-1261).

\section{Methods}

\section{Patients and preoperative evaluation}

We conducted a retrospective review of 36 patients (40 ureters) who underwent minimally invasive UUTRs for various ureteral lesions between April 2019 and March 2020, after obtaining Institutional Review Board approval. The inclusion criterion was ureteral strictures with worsening of the renal pelvic and ureter dilation, progressive decreasing of the differential renal function $<40 \%$. Patients were excluded from the analysis if perioperative or postoperative data were missing. According to the stricture site, length of stricture, affected side and condition of repair materials, different reconstructive techniques under the guidance of our management tactics were chosen. Among them, 18 patients agreed to use off-labeled intraureteral ICG after complete disclosure. All surgeries were performed by two experienced surgeons at our institution in the same period. After grouping the cohorts according to the procedures performed, the demographics, perioperative variables, postoperative complications and follow-up data of the patients were recorded and analyzed between the ICG and non-ICG groups. The study was conducted in accordance with the Declaration of Helsinki (as revised in 2013) and approved by the Institutional Ethics Committee of Peking University First Hospital (2019-134) and individual consent for this retrospective analysis was waived. Obstruction of the ureter was evaluated by CT urography (CTU), retrograde/ antegrade ureteropyelography, ureteroscopy, cystography, and/or a nuclear renal scan. All patients opted for UUTRs after discussion on all available treatment strategies.

\section{ICG preparation and administration}

ICG was prepared by dissolving $25 \mathrm{mg}$ sterile ICG (Dandong Yichuang Pharmaceutical Co., Ltd., Liaoning, China) in $10 \mathrm{~mL}$ distilled water. The $5 \mathrm{~F}$ ureteral catheter was inserted into the diseased ureter and the stricture was located by retrograde pyelography. Then $10 \mathrm{~mL}$ of ICG was injected into the lumen in a retrograde manner through the ureteral catheter, which was placed higher or lower than the level of stenosis. In 14 patients with nephrostomy, $10 \mathrm{~mL}$ of ICG was injected in an antegrade manner into the lumen. Then we clamped the nephrostomy tube and/or ureteral catheter immediately after injection to maximize the retention of the ICG in the ureter. Ureteral catheters were secured to the 16 French Foley catheters.

\section{UUTRs using NIRF imaging}

With the Storz laparoscopic system (Storz GmbH, Tuttlingen, Germany), the fluorescent tracer was detected as a 

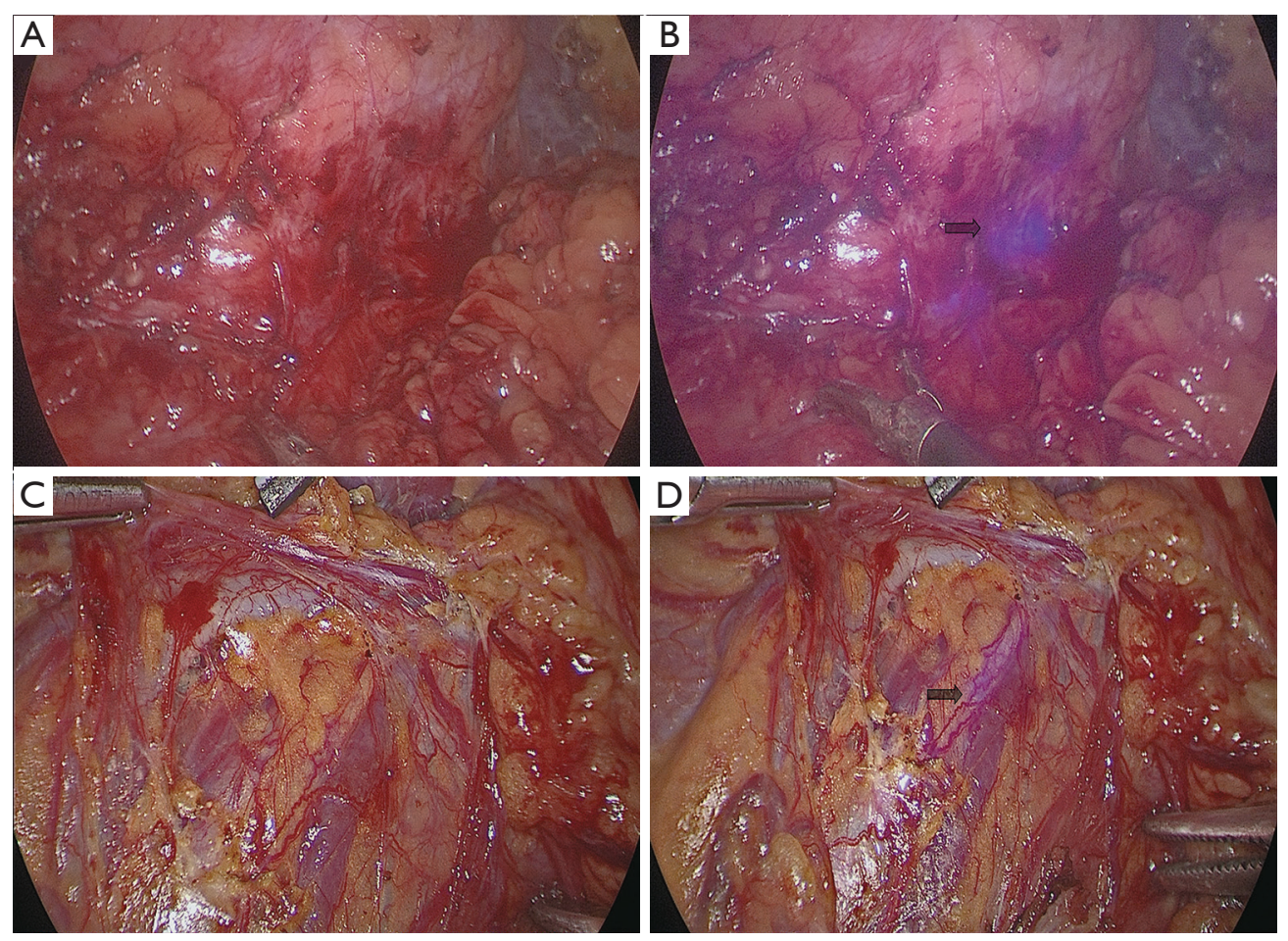

Figure 1 Intraoperative assessment of ureter. (A) Ureteral identification in the white-light view during right ileal ureter replacement; (B) ureteral identification under near-infrared fluorescence (NIRF) during right ileal ureter replacement; (C) ureteral identification in the whitelight view during right ureterolysis; (D) ureteral identification under near-infrared fluorescence (NIRF) during right ureterolysis.

purple color using the NIRF modality, which provides a system for collecting both fluorescent light and white light in the visible light emission range. We used ICG with NIRF imaging in all laparoscopic UUTR procedures to help in objective assessment of ureteral identification and tissue perfusion during dissection. UUTR techniques were used as we previously reported included ileal ureter replacement (7), standardized dismembered pyeloplasty (8), ureteral reimplantation (9), lingual mucosal onlay graft, and appendiceal onlay flap ureteroplasty, ureterolysis, and ureteroureterostomy (10). The principle of UUTRs includes a tension-free, watertight, wellperfused anastomosis $(11,12)$.

The feature of NIRF light modality can be toggled on and off by simultaneous activation of the finger clutch and the camera foot pedal. Under NIRF, the ureter injected with ICG emits purple fluorescence, which can facilitate improved assessment of the ureter (Figure 1). Ureter dissection was performed in the diseased segment along the circumference of the affected area. Attention was paid to avoid direct grasping or manipulation of the ureter to prevent interruption of its fragile blood supply. After ureteral dissection was completed, the NIRF technology was used to accurately locate the site of stenosis. The edge of the ureteral stricture was recognized as a healthy ureter emitting purple fluorescence, while the diseased ureter and inanimate tissue remained unfluoresced or poorly fluoresced (Figure 2). The diseased ureteral segment was then carefully removed using laparoscopic scissors.

\section{Perioperative evaluation and analysis}

We calculated the intraoperative time to identification of the ureter (TIU) and the time to define the stricture (TDS), TIU was defined as the time from the entry of endoscopic instruments into the operative field to the identification of ureter and TDS was defined as the time from the entry of endoscopic instruments into the operative field to the definition of stricture. The estimated blood loss (EBL) and length of postoperative hospital stay (LPHS) were also calculated. Stents were removed at an average time of 8 weeks postoperatively. UUTRs were assessed postoperatively for radiological success (a patent ureter and alleviation of hydronephrosis) and clinical success (absence of symptoms, such as relief of recurrent flank 

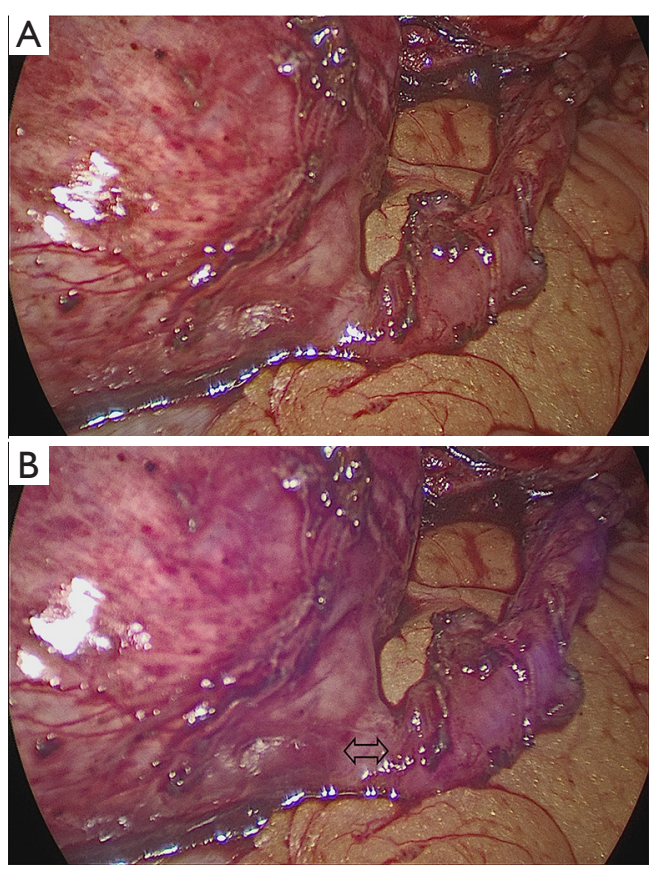

Figure 2 Localization of a ureteral stricture and perfusion. (A) Localization of ureter stricture in white-light view; (B) localization of ureter stricture under NIRF, the nonviable ureter does not fluoresce (arrow), whereas the healthy ureter emits purple fluorescence.

pain and absence of recurrent urinary tract infection). Functional cine magnetic resonance urography (MRU) was performed at 3 months postoperatively (13). Patients were generally arranged for follow-up every 3 months, and it included the following: clinical assessment, renal function (estimated glomerular filtration rate and creatinine), and renal ultrasonography. Perioperative complications were categorized according to the Clavien-Dindo classification system (14).

\section{Statistical analysis}

The data were compared by the nonparametric 2-sample median test. Analyses were performed using SAS software (SAS Institute, Cary, NC, USA). Nominal variables were analyzed by Fisher's exact test or Pearson's chi-squared test, and continuous variables were analyzed by paired t-test. A $\mathrm{P}<0.05$ was considered statistically significant.

\section{Results}

Of the 36 patients, 18 patients (20 ureters) received ICG and 18 patients (20 ureters) did not receive it. Baseline characteristics are shown in Table 1.

According to the location and length of ureteral stenosis, we chose different UUTR methods. In the ICG group, 18 patients (20 ureters) were included; $3 / 20(15.0 \%), 2 / 20$ (10.0\%), $9 / 20(45.0 \%)$, and $2 / 20(10.0 \%)$ ureters were subjected to pyeloplasty, ureterovesical reimplantation, ileal ureter replacement, and lingual mucosal onlay graft or appendiceal onlay flap ureteroplasty, respectively. In the non-ICG group, 18 patients (20 ureters) were included; $8 / 20(40.0 \%), 2 / 20(10.0 \%), 8 / 20$ (40.0\%), and $1 / 20$ $(5.0 \%)$ ureters were subjected to pyeloplasty, ureterovesical reimplantation, ileal ureter replacement, and lingual mucosal onlay graft or appendiceal onlay flap ureteroplasty, respectively. There was no statistically significant difference in age, sex, body mass index (BMI), and location and cause of stenosis between the two groups of patients.

Perioperative outcomes and follow-up data are shown in Table 2. There were no intraoperative or postoperative complications caused by intraureteral administration of ICG, such as anaphylactic shock and cardiorespiratory arrest (15). The intraoperative TIU in the ICG group was shorter than that in the non-ICG group (mean $20.9 v s .30 .0$ min, $\mathrm{P}=0.03$ ). Similar results were observed for the comparison of LPHS (mean 11.1 vs. 16.6 days, $\mathrm{P}=0.017$ ). However, the TDS and EBL in the ICG group were lower than those in the ICG group, but there was no statistically significant difference between these groups (mean time 43.0 vs. 55.4 min, $\mathrm{P}=0.14$; mean EBL 88.3 vs. 91.7 min, $\mathrm{P}=0.22$ ), respectively.

The mean follow-up period was 9.8 months in the ICG group and 12.2 months in the non-ICG group. During the follow-up period, the procedures for all patients were considered to be clinically and radiologically successful. In the non-ICG group, postoperative complications occurred in 4 cases (4/18), including 1 case of incomplete ileus with urinary tract infection, 1 case of incomplete ileus with wound infection, and 2 case of incomplete ileus, all of which were Clavien Grade $2-3$. All 4 cases were cured by conservative therapy. Urinary tract infection (Clavien Grade 1-2), occurred in 3 patients of the ICG group after removal of ureteral stents who were postoperatively managed uneventfully with oral antibiotics.

\section{Discussion}

In the operation of UUTRs, quick and safe identification of the position of the ureter and stenosis, accurate removal of the ureteral stricture, preservation of viable ureter as much as possible to make allowance for a tension-free anastomosis 
Table 1 Baseline characteristics of UUTRs

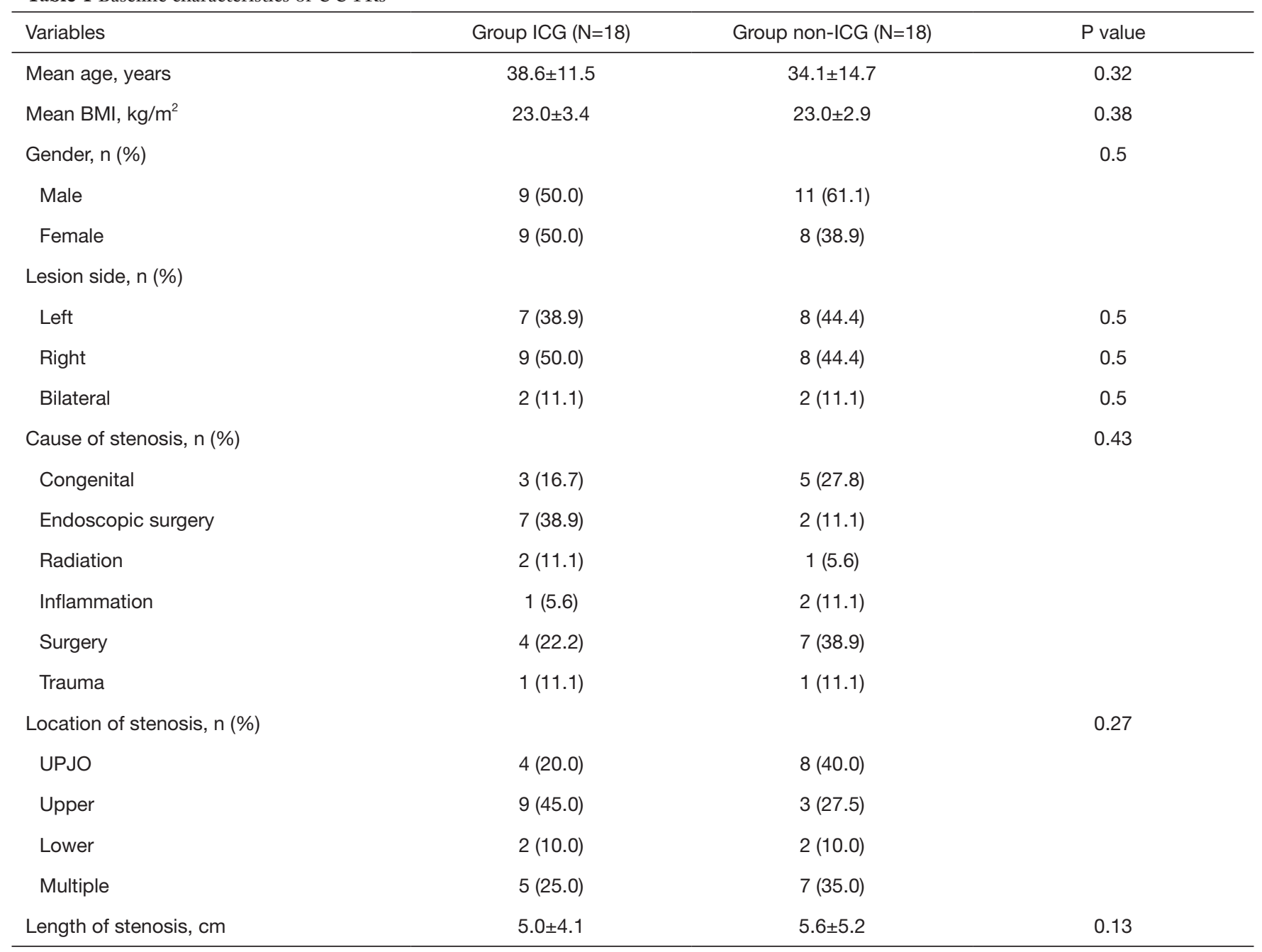

UUTRs, upper urinary tract reconstructions; ICG, indocyanine green; BMI, body mass index; UPJO, ureteropelvic junction obstruction.

are the key points of the operation.

Intraoperative visualization of the ureter embedded in the surrounding tissue is a challenge, especially in patients with secondary UUTRs or high obesity level. Iodine contrast media can be used for intravenous, retrograde or anterograde approaches combined with X-ray fluoroscopy which may expose patients and surgeons to ionizing radiation. Dyes such as methylene blue and indigo carmine can be used for retrograde or anterograde approaches, but it is hard to distinguish whether the ureter is embedded in the tissue and the contrast is poor (16). Under some circumstances, a ureteral stent in combination with an illuminated catheter or palpation (17) can be used for surgical guidance (18). However, illuminated catheters are expensive, and stenting may be difficult and may result in complications (19). In recent years, with the increase in robotic and laparoscopic surgery, these surgical principles are particularly difficult to achieve due to the absence of tactile feedback. In the presence of obliterated dissection planes, inflammation, and fibrosis, which are usually accompanied by pathological changes in the ureter, the performance of UUTRs in maintaining these principles seems more challenging. Thus, a technique that enhances the ability to recognize the ureter and visually differentiate healthy ureters from diseased tissues is the most important (20).

The most extensively studied fluorescent tracer to date is ICG, which can be used as part of a NIRF imaging system, allowing surgeons to switch between fluorescence enhanced 
Table 2 Perioperative results of UUTRs

\begin{tabular}{lcc}
\hline Variables & Group ICG (N=18) & Group non-ICG (N=18) \\
\hline Reconstruction techniques, $n(\%)$ & $3(15.0)$ & - \\
Pyeloplasty & $9(45.0)$ & $8(40.0)$ \\
Ileal ureter replacement & $4(20.0)$ & $1(5.0)$ \\
Lingual mucosal onlay graft or appendiceal onlay flap ureteroplasty & $2(10.0)$ & $2(10.0)$ \\
Ureterovesical reimplantation & $2(10.0)$ & $1(5.0)$ \\
Ureteroureterostomy & $20.9 \pm 11.7$ & $30.0 \pm 14.6$ \\
Mean time of ureter identification, min & $43.0 \pm 27.9$ & 0.03 \\
Mean time of stricture definition, min & $88.3 \pm 75.4$ & 0.14 \\
Mean estimated blood loss, mL & $11.1 \pm 3.0$ & $95.4 \pm 18.6$ \\
Mean post-operation hospital stay, day & $3.8 \pm 1.4$ & $9.7 \pm 46.2$ \\
Median follow-up period, months & $3(16.7)$ & $16.6 \pm 10.0$ \\
Postoperative complications, $n$ (\%) & & $6.2 \pm 2.4$ \\
\hline
\end{tabular}

UUTRs, upper urinary tract reconstructions; ICG, indocyanine green.

views and standard white light in real time (21). ICG also has the ability to penetrate the tissue, and it has a high signalto-noise ratio and an excellent safety profile; thus, it is very suitable as a real-time contrast agent during surgery (3). With the emergence of NIRF in the last 5-6 years, ICG has achieved wide applicability in many fields of surgery: its clinical application has been reported in sentinel lymph node biopsy (SLNB) for stomach cancer, melanoma, breast cancer, and other cancers, in intraoperative tumor imaging, and in intraoperative microvascular free flap angiography during reconstructive surgery (22-25). It has been reported in the urological literature that ICG is helpful in distinguishing the tumor from normal renal parenchyma (26) and in selective renal clamping (27), in guiding sentinel lymph node dissection during radical prostatectomy (28), and in improving tumor localization in partial adrenalectomy (29).

In the past decade, ICG has been utilized as a realtime contrast agent in UUTRs to assist in structural identification, perfusion assessment of ureteral strictures, and delineation of the anastomotic stricture margins (30). Lee et al. reported that intraurethral injection of ICG during ureteroureterostomy can locate the ureteral stricture and they found that it can be used for ureteral repair (20). In 2014, Sam et al. reported the whole process of intraurethral injection of ICG to identify the ureter to prevent injury in gynecological cases. There were no intraoperative or postoperative adverse reactions that could be attributed to ICG (31). In 2015, Lee et al. described 26 cases of robotic- assisted ureter reconstruction, including pyeloplasty, ureteroureterostomy, ureterolysis, and ureteroneocystostomy. At a mean overall follow-up of 12 months, there were no perioperative complications of the novel intraureteral use of ICG (32).

ICG was initially administered intravenously in ureteral surgery to assess ureteral viability and perfusion. In 2013, Bjurlin et al. reported 43 cases of UUTRs by use of intravenous ICG for evaluating the blood supply of the tissues at both ends of the anastomosis, and they found that the fluorescence of the blood flow was poor, the color was dark; otherwise, the fluorescent color was bright (27). However, one of the restrictions of intravenous ICG administration is that ICG is not limited to the ureter, causing background fluorescence, which prompted the other scientists to try a novel, off-label intraureteral ICG application. The mechanism of distinguishing the activity of the ureteral mucosa by intra-ureteral injection of ICG is not clear. It is possible that the active ureteral mucosal cells have higher protein content and are more likely to bind with ICG; thus, they can be better visualized (30).

However, most of these studies are reports of clinical application, and there is a lack of comparative studies on the application of ICG in UUTRs. This study collected data from patients who underwent UUTRs; there were 18 patients in the ICG group and 18 patients in the nonICG group. There was no statistical difference in the baseline characteristics. However, ICG technology showed 
obvious advantages in terms of locating the ureter and recovery after the operation. The mean time of locating the stricture and EBL were lower in the ICG group than in the non-ICG group; however, there was no statistically significant difference between the two groups. This showed that intraureteral injection of ICG with visualization under NIRF can help the surgeon to locate the ureter, identify the site of ureteral stenosis, determine the length of the stricture as soon as possible, and avoid injury to the surrounding tissues and organs; thus, it can reduce the intraoperative complications. It is also beneficial for rapid recovery of patients.

We used a quantitative method to investigate the effectiveness of ICG in surgical navigation during UUTRs for the first time. The ureter identification in the ICG group took less of the time than that in the non-ICG group $(\mathrm{P}=0.011)$. Although the identification of ureter and stricture is only a small part of the whole procedure, it may be the most challenging step in UUTRs, which will affect the outcome of surgery. And we found ICG navigation indeed promote the critical procedure especially in complicated situations and messy anatomical structures: to identify the ureter and stricture. And then, the following procedure of cutting and anastomosis steps can be regarded as routine. On the one hand, it can help the operator to continuously switch from conventional light to near infrared fluorescence during resection to determine ureteric vascularity and the extent of ureteral excision, so as to resect the tissues with poor blood supply to ensure adequate blood supply at both ends of the anastomosis. Further, it can also preserve more blood supply to the tissue to ensure tension-free anastomosis. At a follow-up of approximately 1 year, there were no cases of ureteral stricture in both the groups. Previously published studies suggest that, the use of ICG fluorescence may decrease the incidence of ureteral anastomotic strictures. Thus, a larger sample size and longer follow-up time are needed to verify whether the use of indocyanine decreased the occurrence of anastomotic strictures.

Our study compared the perioperative and follow-up outcomes between the ICG group and the non-ICG group in patients with lesions of the UUT, in order to demonstrate the superiority where ICG was utilized for assessing the ureter comparing it with a cohort where ICG was not used. Firstly, we used intraureteral ICG administration; unlike intravenous ICG use resulting in background fluorescence, only the urinary tract emitted fluorescence. Overall, it was easy to identify a clear anatomical path along the ureter. Our technique minimizes systemic ICG exposure compared with intravenous injection, which reduces the risk of anaphylactic reactions. Secondly, by integrating intraoperative NIFR imaging into the laparoscopic system, providing the technical advantages of minimally invasive surgery, surgeons can identify the anatomy of the pelvis and ureter in real time and perform the dissection meticulously.

Although the technique facilitates UUTRs, there are several limitations to this study. Firstly, it is a retrospective study with a relatively small sample size and short postoperative follow-up time. Thus, there is an urgent need for well-designed randomized controlled trials to quantify the improvement in results after ICG procedures. Secondly, our technique involving the off-label use of ICG is limited to 18 UUTRs, and we did not observe any adverse effects. In order to evaluate the ultimate safety of intraluminal ureteral ICG injection, it is necessary to conduct further studies with a large number of samples and a long-term follow-up. In addition, it is not clear how intraluminal ICG helps to differentiate healthy ureters from diseased ureters. In order to clarify the exact mechanism, our institution is currently conducting a biomolecular and pathological research. Nevertheless, our technique is particularly helpful in the obliterated dissection planes, fibrotic encasement, and setting of inflammation. Therefore, it is necessary to further study and evaluate the application of this technique in urology, gynecology, and general surgery, which may be associated with the risk of accidental ureteral injury.

\section{Conclusions}

Intraureteral ICG injection and subsequent visualization under NIRF help to identify the ureter quickly and accurately and precisely locate the proximal and distal narrow edges of the ureter, thereby contributing to UUTRs. The technique is safe, easy to perform, and repeatable in UUTRs.

\section{Acknowledgments}

Funding: None.

\section{Footnote}

Reporting Checklist: The authors have completed the STROBE reporting checklist. Available at http://dx.doi. org/10.21037/tau-20-1261

Data Sharing Statement: Available at http://dx.doi. org/10.21037/tau-20-1261. 
Conflicts of Interest: All authors have completed the ICMJE uniform disclosure form (available at http://dx.doi. org/10.21037/tau-20-1261). Dr. XL serves as an unpaid editorial board member of Translational Andrology and Urology currently. The authors have no other conflicts of interest to declare.

Ethical Statement: The authors are accountable for all aspects of the work in ensuring that questions related to the accuracy or integrity of any part of the work are appropriately investigated and resolved. The study was conducted in accordance with the Declaration of Helsinki (as revised in 2013) and approved by the Institutional Ethics Committee of Peking University First Hospital (2019-134) and individual consent for this retrospective analysis was waived.

Open Access Statement: This is an Open Access article distributed in accordance with the Creative Commons Attribution-NonCommercial-NoDerivs 4.0 International License (CC BY-NC-ND 4.0), which permits the noncommercial replication and distribution of the article with the strict proviso that no changes or edits are made and the original work is properly cited (including links to both the formal publication through the relevant DOI and the license). See: https://creativecommons.org/licenses/by-nc-nd/4.0/.

\section{References}

1. Marien T, Bjurlin MA, Wynia B, et al. Outcomes of robotic-assisted laparoscopic upper urinary tract reconstruction: 250 consecutive patients. BJU Int 2015;116:604-11.

2. Hyams ES, Mufarrij PW, Stifelman MD. Robotic renal and upper tract reconstruction. Curr Opin Urol 2008;18:557-63.

3. Alander JT, Kaartinen I, Laakso A, et al. A review of indocyanine green fluorescent imaging in surgery. Int J Biomed Imaging 2012;2012:940585.

4. Bates AS, Patel VR. Applications of indocyanine green in robotic urology. J Robot Surg 2016;10:357-9.

5. Polom W, Markuszewski M, Rho YS, et al. Usage of invisible near infrared light (NIR) fluorescence with indocyanine green (ICG) and methylene blue (MB) in urological oncology. Part 1. Cent European J Urol 2014;67:142-8.

6. Ahmadi N, Ashrafi AN, Hartman N, et al. Use of indocyanine green to minimise uretero-enteric strictures after robotic radical cystectomy. BJU Int 2019;124:302-7.

7. Zhong W, Hong P, Ding G, et al. Technical considerations and outcomes for ileal ureter replacement: a retrospective study in China. BMC Surg 2019;19:9.

8. Hong P, Ding G, Zhu D, et al. Head-to-Head Comparison of Modified Laparoscopic Pyeloplasty and Robot-Assisted Pyeloplasty for Ureteropelvic Junction Obstruction in China. Urol Int 2018;101:337-44.

9. Zhong W, Yao L, Cui H, et al. Laparoscopic ureteral reimplantation with extracorporeal tailoring and direct nipple ureteroneocystostomy for adult obstructive megaureter: long-term outcomes and comparison to open procedure. Int Urol Nephrol 2017;49:1973-8.

10. Wang J, Xiong S, Fan S, et al. Appendiceal Onlay Flap Ureteroplasty for the Treatment of Complex Ureteral Strictures: Initial Experience of Nine Patients. J Endourol 2020;34:874-81.

11. Palese MA, Stifelman MD, Munver R, et al. Robotassisted laparoscopic dismembered pyeloplasty: a combined experience. J Endourol 2005;19:382-6.

12. Mufarrij PW, Woods M, Shah OD, et al. Robotic dismembered pyeloplasty: a 6-year, multi-institutional experience. J Urol 2008;180:1391-6.

13. Zhu WJ, Ma MM, Zheng MM, et al. Cine magnetic resonance urography for postoperative evaluation of reconstructive urinary tract after ileal ureter substitution: initial experience. Clin Radiol 2020;75:480.e1-480.e9.

14. Clavien PA, Barkun J, de Oliveira ML, et al. The ClavienDindo classification of surgical complications: five-year experience. Ann Surg 2009;250:187-96.

15. Speich R, Saesseli B, Hoffmann U, et al. Anaphylactoid reactions after indocyanine-green administration. Ann Intern Med 1988;109:345-6.

16. Tanaka E, Ohnishi S, Laurence RG, et al. Real-time intraoperative ureteral guidance using invisible nearinfrared fluorescence. J Urol 2007;178:2197-202.

17. Chahin F, Dwivedi AJ, Paramesh A, et al. The implications of lighted ureteral stenting in laparoscopic colectomy. JSLS 2002;6:49-52.

18. Bothwell WN, Bleicher RJ, Dent TL. Prophylactic ureteral catheterization in colon surgery. A five-year review. Dis Colon Rectum 1994;37:330-4.

19. Wood EC, Maher P, Pelosi MA. Routine use of ureteric catheters at laparoscopic hysterectomy may cause unnecessary complications. J Am Assoc Gynecol Laparosc 1996;3:393-7.

20. Lee Z, Simhan J, Parker DC, et al. Novel use of indocyanine green for intraoperative, real-time 
localization of ureteral stenosis during robot-assisted ureteroureterostomy. Urology 2013;82:729-33.

21. van den Berg NS, van Leeuwen FW, van der Poel HG. Fluorescence guidance in urologic surgery. Curr Opin Urol 2012;22:109-20.

22. Marshall MV, Rasmussen JC, Tan IC, et al. Near-Infrared Fluorescence Imaging in Humans with Indocyanine Green: A Review and Update. Open Surg Oncol J 2010;2:12-25.

23. Zelken JA, Tufaro AP. Current Trends and Emerging Future of Indocyanine Green Usage in Surgery and Oncology: An Update. Ann Surg Oncol 2015;22 Suppl 3:S1271-83.

24. Loutzidou L, Symeonidis S, Ioannidis O, et al. The Clinical Use of Indocyanine Green as a Near-Infrared Fluorescent Contrast Agent for Image-Guided Laparoscopic Surgery in Oncological Patients. Eur J Surg Oncol 2020;46:e160.

25. Sevick-Muraca EM. Translation of near-infrared fluorescence imaging technologies: emerging clinical applications. Annu Rev Med 2012;63:217-31.

26. Tobis S, Knopf JK, Silvers CR, et al. Near infrared fluorescence imaging after intravenous indocyanine green: initial clinical experience with open partial nephrectomy

Cite this article as: Zhu W, Xiong S, Wu Y, Zhang D, Huang C, Hao H, Zhang L, Yang K, Zhang P, Zhu H, Li X, Zhou $\mathrm{L}$. Indocyanine green fluorescence imaging for laparoscopic complex upper urinary tract reconstructions: a comparative study. Transl Androl Urol 2021;10(3):1071-1079. doi: 10.21037/ tau-20-1261 for renal cortical tumors. Urology 2012;79:958-64.

27. Bjurlin MA, Gan M, McClintock TR, et al. Near-infrared fluorescence imaging: emerging applications in robotic upper urinary tract surgery. Eur Urol 2014;65:793-801.

28. van der Poel HG, Buckle T, Brouwer OR, et al. Intraoperative laparoscopic fluorescence guidance to the sentinel lymph node in prostate cancer patients: clinical proof of concept of an integrated functional imaging approach using a multimodal tracer. Eur Urol 2011;60:826-33.

29. Manny TB, Pompeo AS, Hemal AK. Robotic partial adrenalectomy using indocyanine green dye with nearinfrared imaging: the initial clinical experience. Urology 2013;82:738-42.

30. Lee Z, Sterling ME, Keehn AY, et al. The use of indocyanine green during robotic ureteroenteric reimplantation for the management of benign anastomotic strictures. World J Urol 2019;37:1211-6.

31. Siddighi S, Yune JJ, Hardesty JJAJoO, et al. Indocyanine green for intraoperative localization of ureter. Am J Obstet Gynecol 2014;211:436.e1-2.

32. Siddighi S, Yune JJ, Hardesty J, et al. Use of indocyanine green during robot-assisted ureteral reconstructions. Eur Urol 2015;67:291-8. 